\title{
Weyl gauge symmetry and its spontaneous breaking in the standard model and inflation
}

\author{
D. M. Ghilencea ${ }^{1, *}$ and Hyun Min Lee $e^{2,3, \dagger}$ \\ ${ }^{1}$ Department of Theoretical Physics, National Institute of Physics and Nuclear Engineering (IFIN), \\ Bucharest 077125, Romania \\ ${ }^{2}$ Department of Physics, Chung-Ang University, Seoul 06974, Korea \\ ${ }^{3}$ School of Physics, Korea Institute for Advanced Study, Seoul 02455, Korea
}

(Received 25 February 2019; published 6 June 2019)

\begin{abstract}
We discuss the local (gauged) Weyl symmetry and its spontaneous breaking and apply it to model building beyond the standard model (SM) and inflation. In models with nonminimal couplings of the scalar fields to the Ricci scalar that are conformal invariant, the spontaneous generation by a scalar field(s) vacuum expectation value of a positive Newton constant demands a negative kinetic term for the scalar field or vice versa. This is naturally avoided in models with additional Weyl gauge symmetry. The Weyl gauge field $\omega_{\mu}$ couples to the scalar sector but not to the fermionic sector of a SM-like Lagrangian. The field $\omega_{\mu}$ undergoes a Stueckelberg mechanism and becomes massive after "eating" the (radial mode) would-be Goldstone field (dilaton $\rho$ ) in the scalar sector. Before the decoupling of $\omega_{\mu}$, the dilaton can act as an UV regulator and maintain the Weyl symmetry at the quantum level, with relevance for solving the hierarchy problem. After the decoupling of $\omega_{\mu}$, the scalar potential depends only on the remaining (angular variables) scalar fields, which can be the Higgs field, inflaton, etc. We show that a successful inflation is then possible with one of these scalar fields identified as the inflaton. While our approach is derived in the Riemannian geometry with $\omega_{\mu}$ introduced to avoid ghosts, the natural framework is that of Weyl geometry, which for the same matter spectrum is shown to generate the same Lagrangian, up to a total derivative.
\end{abstract}

DOI: 10.1103/PhysRevD.99.115007

\section{INTRODUCTION}

In this paper, we discuss the Weyl gauge symmetry and its spontaneous breaking together with its implications for model building beyond the standard model (SM) and for inflation.

One phenomenological motivation relates to the observation that the SM with a Higgs mass parameter set to zero has a classical scale symmetry [1]. If this symmetry is preserved at the quantum level by (a scale-invariant) UV regularization as in [2-7] and is broken spontaneously only, it can naturally protect at the quantum level a hierarchy of fields vacuum expectation values (VEVs) of the theory $[3,6,8-10]$. The hierarchy we refer to is that between the Higgs field VEV (electroweak scale) and that of new physics represented by the VEV of the flat direction (dilaton) associated with global scale symmetry breaking. Such

\footnotetext{
*dumitru.ghilencea@cern.ch

hminlee@cau.ac.kr
}

Published by the American Physical Society under the terms of the Creative Commons Attribution 4.0 International license. Further distribution of this work must maintain attribution to the author(s) and the published article's title, journal citation, and DOI. Funded by SCOAP. hierarchy of VEVs can be generated by a classical hierarchy of the dimensionless couplings of the theory $[11,12]$.

A proper study of the hierarchy problem, based on the above idea, demands including gravity and generating spontaneously the Planck scale $\left(M_{p}\right)$. This can be done in Brans-Dicke-Jordan theories of gravity [13] via a nonminimal coupling between a scalar field(s) and the scalar curvature $(R)$, when this field(s) develops a nonzero VEV. However, demanding the theory be conformal invariant and spontaneous-only breaking of the conformal symmetry leads to a negative kinetic term for the corresponding scalar field, a nuisance that is often quietly glided over. This problem is automatically avoided in models with Weyl gauge symmetry $[14,16]$ and motivated our study of this symmetry in Secs. II and III.

The Weyl gauge symmetry is the natural extension for conformal-invariant models, e.g., [13-32]; the conformal transformation of the metric is extended by the associated gauge transformation of a Weyl gauge field $\left(\omega_{\mu}\right)$, which is of geometric origin. Section III discusses how $\omega_{\mu}$ undergoes a Stueckelberg mechanism and becomes massive by eating the would-be Goldstone field (dilaton $\rho$ ); here, the dilaton is the radial direction in the field space of scalar fields $\left(\phi_{j}\right)$ of different nonminimal couplings $\xi_{j}$ to $R$. The Weyl gauge symmetry is then spontaneously broken and 
there are no negative kinetic terms in the theory. The vacuum expectation value $\langle\rho\rangle$ of the flat direction (dilaton) controls the mass of $\omega_{\mu}$ and $M_{p}$. After $\omega_{\mu}$ decouples, the potential depends only on the remaining angular variables' scalar fields, which can account for the Higgs field, inflaton, etc. ${ }^{1}$ Our analysis extends previous studies [19-23] to multiple scalar fields $\left(\phi_{j}\right)$ and different nonminimal couplings $\left(\xi_{j}\right)$.

We show (Sec. III D) how, prior to this symmetry breaking, the dilaton can enforce an UV regularization of the quantum corrections that keeps manifest the Weyl symmetry. In Weyl-invariant models, the dilaton replaces the subtraction scale, thus maintaining this symmetry at the quantum level [2-7], after which it is eaten by $\omega_{\mu}$ and disappears from the spectrum. One is left with the potential for angular variables' fields (e.g., Higgs field, etc.). This is relevant for the hierarchy problem in Weyl-symmetric theories.

While our analysis (Sec. II B) is formulated in Riemannian geometry (RG) extended by the Weyl gauge symmetry, the natural framework for this study is Weyl conformal geometry (WG) [14-16]. In the RG case, imposing the Weyl symmetry to avoid generating ghosts leads to a SM-like Lagrangian with the corresponding current $K_{\mu}=\partial_{\mu} K$, where $K_{\mu}$ interacts with the field $\omega_{\mu}$ and $K=\rho^{2}$. We show that this Lagrangian is identical, up to a total derivative term, to the simplest Lagrangian one can build in the Weyl geometry for the same set of matter fields, using the curvature scalar and curvature tensors of WG (Sec. II C). This equivalence is an interesting result that follows from the relation between $R$ computed in Riemannian geometry and its counterpart $\tilde{R}$ computed in Weyl geometry.

We also verify that, in the Lagrangian $\mathcal{L}$ of the SM endowed with Weyl gauge symmetry, unlike the Higgs sector, gauge bosons and fermions do not couple to $\omega_{\mu}$ [22] [except a possible kinetic mixing of $\omega_{\mu}$ to $U(1)_{Y}$ ]. $\mathcal{L}$ can be used for further phenomenological studies of the Weyl gauge symmetry.

For the case of two scalar fields present with nonminimal couplings, after the Weyl field $\omega_{\mu}$ decouples, the potential depends only on the angular field $\theta$ and becomes constant for large $\tan \theta$. We show that successful inflation is then possible, in which the field $\theta$ is playing the role of the inflaton. This is another result of this work, discussed in Sec. IV. Our conclusions are presented in Sec. V.

\footnotetext{
${ }^{1}$ The mechanism of the Weyl gauge symmetry breaking used here differs from that in [23], where a complex scalar is considered rather the (real) dilaton and a Coleman-Weinberg mechanism (unitary gauge) is used (instead of a Stueckelberg mechanism), which breaks explicitly the Weyl symmetry by UV regularization. An explicit (classical) breaking was also considered in [25] for one scalar field case.
}

\section{IMPLICATIONS OF WEYL GAUGE SYMMETRY}

We review how models invariant under conformal transformations become ghost-free while generating spontaneously a positive Newton constant, when a Weyl gauge transformation is added. The Lagrangian so obtained is then shown to be equivalent to that derived in Weyl geometry, up to a total derivative; a SM-like model with this symmetry is also constructed.

\section{A. Weyl symmetry or how to obtain a Lagrangian without ghosts}

Consider a (local) conformal transformation of the metric ${ }^{2}$ and of a scalar field $\phi$ and a fermion $\psi$ as follows:

$$
\begin{aligned}
g_{\mu \nu} & \rightarrow g_{\mu \nu}^{\prime}=e^{2 \alpha(x)} g_{\mu \nu}, \\
\phi & \rightarrow \phi^{\prime}=e^{-\alpha(x) \Delta_{s}} \phi, \quad \psi \rightarrow \psi^{\prime}=e^{-\alpha(x) \Delta_{f}} \psi .
\end{aligned}
$$

Then $g^{\mu \nu \prime}=e^{-2 \alpha(x)} g^{\mu \nu}$ and $\sqrt{g^{\prime}}=e^{4 \alpha(x)} \sqrt{g}$ with $g=$ $\left|\operatorname{det} g_{\mu \nu}\right|$. Here $\Delta_{s}=1$ and $\Delta_{f}=3 / 2$.

We would like to generate the Planck scale spontaneously, from the VEV of a scalar field $\phi$. To this purpose, one uses that the Lagrangian

$$
L_{1}^{ \pm}= \pm \sqrt{g} \frac{\xi}{2}\left\{\frac{1}{6} \phi^{2} R+g^{\mu \nu} \partial_{\mu} \phi \partial_{\nu} \phi\right\}
$$

is invariant under transformation (1). ${ }^{3} \xi$ is the nonminimal coupling and we assume $\xi>0$.

Then one is facing the following issue. To generate the Einstein term

$$
L_{E}=-\frac{1}{2} \sqrt{g} M_{p}^{2} R
$$

after spontaneous breaking of conformal symmetry from a $\mathrm{VEV}$ of $\phi$ from the first term in (2), one must take the minus sign in front of (2); that means a negative kinetic term for $\phi$ (ghost) is present in the theory, which may not be acceptable. Alternatively, a positive kinetic term leads to $M_{p}^{2}<0$. One usually sets $M_{p}=\langle\phi\rangle$ (gauge fixing the Planck scale) and the ghost presence is then ignored. Yet, one cannot have the benefit of conformal symmetry but ignore this side effect, therefore, we would like to understand its meaning.

To avoid this problem, we associate with transformation (1) that of a (Weyl) vector field $\omega_{\mu}$ [14] which, in light of (1), is of geometric origin

\footnotetext{
${ }^{2}$ Conventions: metric $(+,-,-,-), R_{\mu \nu \sigma}^{\lambda}=\partial_{\nu} \Gamma_{\mu \sigma}^{\lambda}-\partial_{\sigma} \Gamma_{\mu \nu}^{\lambda}+$ $\Gamma_{\nu \rho}^{\lambda} \Gamma_{\mu \sigma}^{\rho}-\Gamma_{\sigma \rho}^{\lambda} \Gamma_{\mu \nu}^{\rho}, R_{\mu \sigma}=R_{\mu \lambda \sigma}^{\lambda}, R=g^{\mu \nu} R_{\mu \nu}$.

${ }^{3}$ To see this, one uses that under Eq. (1) $R$ transforms as $R \rightarrow R^{\prime}=e^{-2 \alpha(x)}\left(R-6 e^{-\alpha(x)} \square e^{\alpha(x)}\right)$.
} 


$$
\omega_{\mu} \rightarrow \omega_{\mu}^{\prime}=\omega_{\mu}-\frac{2}{q} \partial_{\mu} \alpha(x)
$$

then consider adding the kinetic term below, with a suitable normalization coefficient

$$
L_{2}=\frac{1}{2}(1+\xi) \sqrt{g} g^{\mu \nu} \tilde{D}_{\mu} \phi \tilde{D}_{\nu} \phi, \quad \tilde{D}_{\mu} \equiv \partial_{\mu}-\frac{q}{2} \omega_{\mu}
$$

$L_{2}$ is invariant under (1) and (4) since $\tilde{D}_{\mu} \phi \rightarrow e^{-\alpha} \tilde{D}_{\mu} \phi$, due to the presence of $\omega_{\mu}$. Since $L_{1}^{ \pm}$is also invariant under (1) and (4), the sum $L_{1}^{ \pm}+L_{2}$ is also invariant. Hereafter, we take $L_{1}^{-}$. One has $L_{1}^{-}+L_{2}=(1 / 2) g^{\mu \nu} \partial_{\mu} \phi \partial_{\nu} \phi-$ $(1 / 12) \xi \phi^{2} R+\cdots$, with a canonically normalized kinetic term for $\phi$. Thus, the Planck (mass) ${ }^{2}$ generated by $\left\langle\phi^{2}\right\rangle$ and the kinetic term of $\phi$ can be simultaneously positive. ${ }^{4}$ This is made possible by the additional presence of the Weyl field $\omega_{\mu}$; this is a sufficient condition for the consistency of the theory (absence of ghosts).

\section{B. SM Lagrangian with Weyl gauge symmetry}

We use the above observation about $L_{1}^{-}+L_{2}$ to construct a Lagrangian without ghosts and invariant under Eqs. (1) and (4). For generality, consider a version of $L_{1}^{-}+L_{2}$ with more scalar fields $\phi_{j}$ of nonminimal couplings $\xi_{j}$, then a Weyl-invariant Lagrangian is

$$
\begin{aligned}
L= & \sqrt{g}\left\{-\frac{\xi_{j}}{2}\left[\frac{1}{6} \phi_{j}^{2} R+g^{\mu \nu} \partial_{\mu} \phi_{j} \partial_{\nu} \phi_{j}\right]\right. \\
& \left.+\left(1+\xi_{j}\right) \frac{1}{2} g^{\mu \nu} \tilde{D}_{\mu} \phi_{j} \tilde{D}_{\nu} \phi_{j}-V\left(\phi_{j}\right)\right\} .
\end{aligned}
$$

A summation is understood over repeated index $j=1,2,3, \ldots$. We also added a potential $V\left(\phi_{j}\right)$ for the scalars $\phi_{j}$; given the conformal symmetry, $V$ is a homogeneous function, so

$$
V\left(\phi_{j}\right)=\phi_{k}^{4} V\left(\phi_{j} / \phi_{k}\right), \quad k=\text { fixed }
$$

$L$ can be rewritten as

$$
\begin{aligned}
L= & \sqrt{g}\left\{-\frac{\xi_{j}}{12} \phi_{j}^{2} R+\frac{g^{\mu \nu}}{2}\left(\partial_{\mu} \phi_{j}\right)\left(\partial_{\nu} \phi_{j}\right)\right. \\
& \left.-\frac{q}{4} g^{\mu \nu} \omega_{\mu} K_{\nu}+\frac{q^{2}}{8} K \omega_{\mu} \omega^{\mu}-V\left(\phi_{j}\right)\right\},
\end{aligned}
$$

where

\footnotetext{
${ }^{4}$ This is automatic in Weyl geometry, see Sec. IIC and $[19,22,23]$.
}

$$
K_{\nu}=\partial_{\nu} K, \quad K=\left(1+\xi_{j}\right) \phi_{j}^{2}
$$

$L$ above is invariant under (1) and (4) for all values of $\xi_{j}$, thanks to the $\omega_{\mu}$-dependent terms. $L$ has a positive kinetic term for $\phi_{j}$ and $M_{p}^{2}>0$ when generated by the VEV of $\langle\phi\rangle$ (assuming $\xi_{j}>0$ ). In the absence of the $\omega_{\mu}$-dependent part, $L$ is not conformal (unless $\xi_{j}=-1$ ), but only globally conformal. Unlike in gauge theories, $\omega_{\mu}$ is a vector under a real transformation of the fields $\phi_{j}$ (missing the $i$ factor). The associated current $K_{\mu}$ is nonzero for $\phi_{j}$ reals.

Further, we include a kinetic term for $\omega_{\mu}$ with the usual (pseudo)Riemannian definition

$$
\begin{aligned}
L_{g} & =-\frac{\sqrt{g}}{4} g^{\mu \rho} g^{\nu \sigma} F_{\mu \nu} F_{\rho \sigma}, \quad F_{\mu \nu}=D_{\mu} \omega_{\nu}-D_{\nu} \omega_{\mu}, \\
D_{\mu} \omega_{\nu} & =\partial_{\mu} \omega_{\nu}-\Gamma_{\mu \nu}^{\rho} \omega_{\rho} .
\end{aligned}
$$

$L_{g}$ is invariant under (1) and (4), since the metric part is invariant and $F_{\mu \nu}\left(=\partial_{\mu} \omega_{\nu}-\partial_{\nu} \omega_{\mu}\right)$ is invariant, too. The Riemann connection ${ }^{5} \Gamma_{\mu \nu}^{\rho}$, symmetric under the exchange $\mu \leftrightarrow \nu$, is not invariant under (1).

Finally, one can consider the Weyl-invariant Lagrangian $L_{f}$ for the massless fermions of the theory that transform under (1). $L_{f}$ has the usual form in (pseudo)Riemann space

$$
L_{f}=\sqrt{g} \bar{\psi} i \gamma^{a} e^{\mu}{ }_{a} D_{\mu} \psi, \quad D_{\mu} \psi=\left(\partial_{\mu}+\frac{1}{2} \omega_{\mu}^{a b} \sigma_{a b}\right) \psi,
$$

where $\omega_{\mu}^{a b}=e^{\lambda b}\left(-\partial_{\mu} e_{\lambda}^{a}+e_{\nu}^{a} \Gamma_{\mu \lambda}^{\nu}\right)$ is the spin connection and $\sigma_{a b}=\frac{1}{4}\left[\gamma_{a}, \gamma_{b}\right]$. Note that $g_{\mu \nu}=e_{\mu}{ }^{a} e_{\nu}{ }^{b} \eta_{a b}$ and $e^{\mu}{ }_{a} e_{\nu}{ }^{a}=\delta_{\nu}^{\mu}$. Under a Weyl transformation of the metric (1), the vierbein $e_{\mu}^{a}$ transforms as $e_{\mu}{ }^{a \prime}=e^{\alpha(x)} e_{\mu}{ }^{a}$, while for the spin connection we have $\omega_{\mu}^{a b \prime}=$ $\omega_{\mu}^{a b}+\left(e_{\mu}{ }^{a} e^{\nu b}-e^{\nu a} e_{\mu}{ }^{b}\right) \partial_{\mu} \alpha$. Then it can be shown that $L_{f}$ is invariant under a Weyl gauge transformation [Eqs. (1) and (4)] and there is no coupling of fermions to the gauge field $\omega_{\mu}$.

Regarding the SM gauge fields' kinetic terms $\left(L_{G}\right)$, these are invariant under Weyl gauge symmetry. Indeed, the gauge fields' presence under the covariant derivative that contains $\partial_{\mu}$ shows that these are invariant, since coordinates do not transform under (1). Therefore, there is no coupling between the SM gauge fields and $\omega_{\mu}{ }^{6}$ For example, for the $U(1)_{Y}$ gauge field $A_{\mu}$, the covariant derivative can be written as $D_{\mu} A_{\nu}=\partial_{\mu} A_{\nu}-\Gamma_{\mu \nu}^{\rho} A_{\rho}$. The gauge kinetic terms

\footnotetext{
${ }^{5}$ The Riemann affine connection used here is $\Gamma_{\mu \nu}^{\rho}=$ $(1 / 2) g^{\rho \beta}\left[\partial_{\nu} g_{\beta \mu}+\partial_{\mu} g_{\beta \nu}-\partial_{\beta} g_{\mu \nu}\right]$.

${ }^{6} \mathrm{An}$ exception is a possible kinetic mixing of the field strength of $\omega_{\mu}$ to that of $U(1)_{Y}$ [33].
} 
do not contain the Christoffel symbols because $F_{\mu \nu}=$ $D_{\mu} A_{\nu}-D_{\nu} A_{\mu}=\partial_{\mu} A_{\nu}-\partial_{\nu} A_{\mu}$.

The sum, $\mathcal{L}=L+L_{g}+L_{f}+L_{G}$, is the total SM-like Lagrangian with Weyl gauge symmetry, ${ }^{7}$ which is invariant under (1) and (4). Here $L$ is immediately adapted to accommodate the Higgs doublet of the SM with one of the $\phi_{j}$ fields to account for the Higgs neutral scalar. In conclusion, we have a SM-like Lagrangian that is invariant under (1) and (4).

\section{From Riemann to Weyl conformal geometry}

The presence of the Weyl gauge field in our model in the Riemannian geometry and invariant under (1) and (4) is natural in Weyl's conformal geometry [14,16] (also [15]). Following [16], we write the SM-like Lagrangian with this symmetry directly in Weyl geometry and we verify that it agrees with that of the previous section, built in the Riemannian geometry (with $\omega_{\mu}$ introduced to avoid ghosts).

Weyl geometry is a scalar-vector-tensor theory of gravity and thus provides a generalization (to classes of equivalence) of Brans-Dicke-Jordan scalar-tensor theory [13] and of other conformal-invariant models [18]. It was used for model building $[19,20]$ with renewed recent interest in [22-29] and applications to inflation, see, e.g., [30-32,36-41]. If the Weyl field is set to zero, one obtains (Weyl integrable) models similar to Brans-Dicke-Jordan theory [29].

In Weyl geometry, the curvature scalars and tensors and the connection are different from the Riemannian case where they are induced by the metric alone. In Weyl geometry,

$$
\tilde{\Gamma}_{\mu \nu}^{\rho}=\Gamma_{\mu \nu}^{\rho}+\frac{q}{2}\left[\delta_{\mu}^{\rho} \omega_{\nu}+\delta_{\nu}^{\rho} \omega_{\mu}-g_{\mu \nu} \omega^{\rho}\right],
$$

where $\Gamma_{\mu \nu}^{\rho}$ are the connection coefficients in the Riemannian geometry. Under (1) and (4), the coefficients $\tilde{\Gamma}_{\mu \nu}^{\rho}$ are invariant, as one can easily check. The system is torsion-free. The Riemann tensor in Weyl geometry is then generated by the new $\tilde{\Gamma}_{\mu \nu}^{\rho}$

$$
\tilde{R}_{\mu \nu \sigma}^{\lambda}=\partial_{\nu} \tilde{\Gamma}_{\mu \sigma}^{\lambda}-\partial_{\sigma} \tilde{\Gamma}_{\mu \nu}^{\lambda}+\tilde{\Gamma}_{\nu \rho}^{\lambda} \tilde{\Gamma}_{\mu \sigma}^{\rho}-\tilde{\Gamma}_{\sigma \rho}^{\lambda} \tilde{\Gamma}_{\mu \nu}^{\rho},
$$

and then $\tilde{R}_{\mu \sigma}=\tilde{R}_{\mu \lambda \sigma}^{\lambda}, \tilde{R}=g^{\mu \nu} \tilde{R}_{\mu \nu}$. We can then compute $\tilde{R}$ and find

$$
\begin{aligned}
\tilde{R} & =R-3 q\left[\partial_{\mu} \omega^{\mu}+\frac{1}{2} \omega^{\rho} g^{\lambda \beta} \partial_{\rho} g_{\lambda \beta}\right]-\frac{3}{2} q^{2} \omega^{\mu} \omega_{\mu} \\
& =R-3 q D_{\mu} \omega^{\mu}-\frac{3}{2} q^{2} \omega^{\mu} \omega_{\mu} .
\end{aligned}
$$

\footnotetext{
${ }^{7}$ One could also add a Weyl tensor-squared term to the action, which is invariant under (1), or a quadratic term in the Weyl scalar curvature $\tilde{R}^{2}$, see $[34,35]$ for further details.
}

Then under transformations (1) and (4),

$$
\tilde{R} \rightarrow \tilde{R}^{\prime}=e^{-2 \alpha(x)} \tilde{R} .
$$

As a result

$$
L_{1 w}=-\sqrt{g} \frac{1}{12} \xi_{j} \phi_{j}^{2} \tilde{R}, \quad(\text { sum over } j=1,2 .)
$$

is invariant under combined transformations (1) and (4). This is unlike the Riemannian case of the previous section where the nonminimal coupling term in the action was not invariant.

Further, we can define a kinetic term for $\phi$ in Weyl geometry, invariant under (1) and (4),

$$
L_{2 w}=\frac{1}{2} \sqrt{g} g^{\mu \nu} \tilde{D}_{\mu} \phi_{j} \tilde{D}_{\nu} \phi_{j}-\sqrt{g} V\left(\phi_{j}\right) .
$$

We also have a gauge kinetic term $\left(L_{3 w}\right)$ for $\omega_{\mu}$, now defined by new coefficients $\tilde{\Gamma}$ of (12)

$$
\begin{aligned}
L_{3 w} & =-\frac{\sqrt{g}}{4} g^{\mu \rho} g^{\nu \sigma} F_{\mu \nu} F_{\rho \sigma}, \quad F_{\mu \nu}=\tilde{D}_{\mu} \omega_{\nu}-\tilde{D}_{\nu} \omega_{\mu}, \\
\tilde{D}_{\mu} \omega_{\nu} & =\partial_{\mu} \omega_{\nu}-\tilde{\Gamma}_{\mu \nu}^{\rho} \omega_{\rho} .
\end{aligned}
$$

However, $\tilde{\Gamma}_{\mu \nu}^{\rho}$ are symmetric in $\mu \leftrightarrow \nu$ and also invariant under Weyl transformation Eqs. (1) and (4). Thus, $F_{\mu \nu}$ and $L_{3 w}$ are equal to their counterparts in the previous section [Eq. (10)], so $L_{3 w}=L_{g}$. The same can be said about the SM gauge fields' kinetic terms.

Further, the fermionic Lagrangian is defined with the Weyl connection, as follows:

$$
\begin{aligned}
L_{4 w} & =\sqrt{g} \bar{\psi} i \gamma^{a} e_{a}^{\mu} \tilde{D}_{\mu} \psi, \\
\tilde{D}_{\mu} \psi & =\left(\partial_{\mu}+\frac{1}{2} \tilde{\omega}_{\mu}^{a b} \sigma_{a b}-\frac{3}{4} q \omega_{\mu}\right) \psi,
\end{aligned}
$$

where $\tilde{\omega}_{\mu}^{a b}=e^{\lambda b}\left(-\partial_{\mu} e_{\lambda}{ }^{a}+e_{\nu}{ }^{a} \tilde{\Gamma}_{\mu \lambda}^{\nu}\right)$. However, one shows [22] that $L_{4 w}=L_{f}$ with $L_{f}$ of (11).

Adding together $L_{1 w}, L_{2 w}, L_{3 w}$, and $L_{4 w}$, each of these invariant under (1) and (4), we obtain a total Lagrangian for the case of Weyl geometry. It is interesting to see that this Lagrangian is equal to $L+L_{g}+L_{f}$ of (8), (10), and (11), up to a total derivative term. This follows from the relation

$$
L_{1 w}+L_{2 w}=L+\frac{q}{4} \xi_{j} \partial_{\mu}\left[\sqrt{g} \phi_{j}^{2} \omega^{\mu}\right] .
$$

To show Eq. (20), one uses the relation between $\tilde{R}$ and $R$ of Eq. (14) that relates Weyl and Riemann scalar curvatures and that $\partial_{\lambda} g=g g^{\mu \sigma} \partial_{\lambda} g_{\sigma \mu}$.

Equation (20) shows that our model agrees (for the twofields case) with that in [22] built within Weyl geometry from the onset and following [16]. We thus obtained the 
same Lagrangian in Riemann and Weyl geometry, albeit with different initial motivations. Our motivation for a consistent ghost-free conformal action, with this symmetry broken spontaneously, led us to introduce a gauge transformation and Weyl gauge field associated with (1).

\section{SPONTANEOUS BREAKING OF WEYL GAUGE SYMMETRY}

In this section, we show how the Weyl conformal symmetry of our model is spontaneously broken for one or more scalar fields of nonminimal couplings $\xi_{j}$ to $R$. Then, we show that the (radial mode) would-be Goldstone boson (dilaton $\rho$ ) of the Weyl symmetry decouples from the angular variables' fields, due to a Stueckelberg mechanism for the Weyl gauge field, which becomes massive. Before decoupling, the dilaton can provide a scale-invariant UV regularization for models in which quantum scale invariance is important.

\section{A. One scalar field and the Stueckelberg mechanism for $\omega_{\mu}$}

Let us first show how spontaneous breaking of Weyl symmetry happens for one scalar field $\phi$. Then $L$ of Eq. (8) simplifies (no sum over $j$ ) and we replace $\phi_{j} \rightarrow \phi$, then

$$
K=(1+\xi) \phi^{2}, \quad V=\frac{\lambda}{4 !} \phi^{4},
$$

where $V$ is the only one allowed by the Weyl symmetry. To decouple the scalar field fluctuations from $R$, we go to the Einstein frame by rescaling the metric to

$$
\hat{g}_{\mu \nu}=\Omega g_{\mu \nu}, \quad \Omega=\frac{\xi}{6} \frac{\phi^{2}}{\langle\phi\rangle^{2}} .
$$

Hereafter, a hat on a variable denotes the Einstein frame value of that variable. From Eq. (8) for one field and Eq. (22) we obtain the tensor-scalar part of the Einstein frame Lagrangian as

$$
\begin{aligned}
\hat{L}= & \sqrt{\hat{g}}\left\{-\frac{1}{2}\langle\phi\rangle^{2} \hat{R}+\frac{3}{4}\langle\phi\rangle^{2}\left(\partial_{\mu} \ln \Omega\right)^{2}\right. \\
& \left.+\frac{1}{\Omega}\left[\frac{1}{2}\left(\partial_{\mu} \phi\right)^{2}+\frac{q^{2}}{8} K \omega_{\mu} \omega^{\mu}-\frac{q}{4} \omega^{\mu} K_{\mu}\right]-\frac{V}{\Omega^{2}}\right\},
\end{aligned}
$$

giving

$$
\begin{aligned}
\hat{L}= & \sqrt{\hat{g}}\left[-\frac{1}{2}\langle\phi\rangle^{2} \hat{R}+3\langle\phi\rangle^{2}\left(1+\frac{1}{\xi}\right)\left(\frac{\partial_{\mu} \phi}{\phi}\right)^{2}\right. \\
& +\frac{3}{4} q^{2}\langle\phi\rangle^{2}\left(1+\frac{1}{\xi}\right) \omega_{\mu} \omega^{\mu} \\
& \left.-3 q\langle\phi\rangle^{2}\left(1+\frac{1}{\xi}\right) \omega^{\mu} \partial_{\mu} \ln \phi-\frac{3 \lambda}{2 \xi^{2}}\langle\phi\rangle^{4}\right],
\end{aligned}
$$

where all contractions are with the new metric $\hat{g}_{\mu \nu}$. Finally, we introduce

$$
\omega_{\mu}^{\prime}=\omega_{\mu}-\frac{2}{q} \partial_{\mu} \ln \phi
$$

giving

$\hat{L}=\sqrt{\hat{g}}\left[-\frac{1}{2}\langle\phi\rangle^{2} \hat{R}+\frac{3}{4} q^{2}\langle\phi\rangle^{2}\left(1+\frac{1}{\xi}\right) \omega_{\mu}^{\prime} \omega^{\prime \mu}-\frac{3 \lambda}{2 \xi^{2}}\langle\phi\rangle^{4}\right]$.

As a result, the scalar (dilaton) field $\phi$ is eaten by the Weyl gauge boson $\omega_{\mu}$. The mass of $\omega_{\mu}$ is $m_{\omega}^{2}=$ $\left(3 q^{2} / 2\right)(1+1 / \xi)\langle\phi\rangle^{2}$. Therefore, conformal symmetry is broken spontaneously as in the Stueckelberg formulation for a massive $U(1)$ without a corresponding Higgs mode. The number of degrees of freedom (d.o.f.) remains the same (three): in the Jordan frame we had a real scalar and a massless vector, while in Einstein frame, after breaking there is no scalar field but a massive vector boson. Also note that the gauge kinetic term $L_{g}$ of $\omega_{\mu}$, see Eq. (10), is invariant under (22) and (25). The scalar potential becomes a cosmological constant, $V_{0}=3 \lambda\langle\phi\rangle^{4} /\left(2 \xi^{2}\right)$, in the Einstein frame.

The transformation (25) may be seen as a Weyl gauge transformation (4) with $\alpha=\ln \sqrt{\Omega}$ corresponding to (22). Then the scalar field $\phi$ transforms according to Eq. (1) into

$$
\phi^{\prime}=e^{-\ln \sqrt{\Omega}} \phi=\sqrt{6 / \xi}\langle\phi\rangle,
$$

so $\phi^{\prime}$ is not dynamical anymore. Therefore spontaneous breaking of conformal symmetry fixing the Planck scale (to $\left.M_{p}=\langle\phi\rangle\right)$ and Stueckelberg mechanism are related to a Weyl transformation to a special unitary gauge (gauge fixing).

While we used $\langle\phi\rangle \neq 0$ in the definition of $\Omega$ and subsequent equations, this is actually not needed and an arbitrary mass scale $\mathcal{M}$ can be used instead of $\langle\phi\rangle$, corresponding to a different gauge fixing (and different Planck scale). Indeed, the Stueckelberg mechanism is a rearrangement of the d.o.f. (which does not require $\langle\phi\rangle \neq 0)$. Using an arbitrary $\mathcal{M}$ is consistent with the fact that, for a single scalar field in a Weyl-invariant theory, $\langle\phi\rangle$ cannot be determined from the condition $4 V(\phi)-\phi V^{\prime}(\phi)=0$, which is automatically respected, hence $\langle\phi\rangle$ remains a parameter (unknown). This condition is also related to the conservation of the current $D^{\mu} K_{\mu}=0$, which for a Friedmann-Robertson-Walker metric leads to a constant solution $\langle\phi\rangle[30,31]$ that is not fixed by the theory. 


\section{B. Two scalar fields and the Stueckelberg mechanism for $\omega_{\mu}$}

Let us consider now the more interesting case of two scalar fields in Eq. (8), $(j=1,2)$. Then

$$
K=\left(1+\xi_{1}\right) \phi_{1}^{2}+\left(1+\xi_{2}\right) \phi_{2}^{2}
$$

Since $V$ is a homogeneous function of fields, one can have

$$
V\left(\phi_{1}, \phi_{2}\right)=\frac{\lambda_{1}}{4 !} \phi_{1}^{4}+\frac{\lambda_{12}}{4} \phi_{1}^{2} \phi_{2}^{2}+\frac{\lambda_{2}}{4 !} \phi_{2}^{4} .
$$

In particular, if $3 \lambda_{12}=-\sqrt{\lambda_{1} \lambda_{2}}$, then

$$
V\left(\phi_{1}, \phi_{2}\right)=\frac{\lambda_{1}}{4 !}\left(\phi_{1}^{2}-\frac{\sqrt{\lambda_{2}}}{\sqrt{\lambda_{1}}} \phi_{2}^{2}\right)^{2}
$$

$V$ can also contain terms like $\phi_{1}^{6} / \phi_{2}^{2}$, etc. [6]. The results below are for a general homogeneous function $V\left(\phi_{1,2}\right)$, i.e., it has a flat direction: $V\left(\phi_{1,2}\right)=\left(\phi_{1}^{2}-k_{0} \phi_{2}^{2}\right)^{2} f\left(\phi_{1} / \phi_{2}\right)$, $\left(k_{0}=\right.$ constant $)$.

To decouple $R$ from the fluctuations of $\phi_{1,2}$, we consider a transformation to the Einstein frame. Let us perform a metric rescaling of $L$, Eq. (8), to

$$
\begin{aligned}
\hat{g}_{\mu \nu} & =\Omega g_{\mu \nu}, \quad \Omega=\frac{1}{6 v^{2}}\left(\xi_{1} \phi_{1}^{2}+\xi_{2} \phi_{2}^{2}\right), \\
v^{2} & \equiv\left\langle\xi_{1} \phi_{1}^{2}+\xi_{2} \phi_{2}^{2}\right\rangle .
\end{aligned}
$$

Here $v$ ensures that $\Omega$ is dimensionless. ${ }^{8}$ From Eq. (8) for two fields and with (31), we obtain the corresponding Einstein frame Lagrangian as

$$
\begin{aligned}
\hat{L}= & \sqrt{\hat{g}}\left[-\frac{1}{2} v^{2} \hat{R}+\frac{3}{4} v^{2}\left(\partial_{\mu} \ln \Omega\right)^{2}+\frac{1}{\Omega}\left(\frac{1}{2}\left(\partial_{\mu} \phi_{1}\right)^{2}\right.\right. \\
& \left.\left.+\frac{1}{2}\left(\partial_{\mu} \phi_{2}\right)^{2}+\frac{q^{2}}{8} K \omega_{\mu} \omega^{\mu}-\frac{q}{4} \omega^{\mu} K_{\mu}\right)-\hat{V}\right],
\end{aligned}
$$

where all contractions are with the new metric $\hat{g}_{\mu \nu} ; \Omega, K$, and $\hat{V}$ are functions of $\phi_{1,2}$ with

$$
\hat{V}\left(\phi_{1}, \phi_{2}\right)=\frac{1}{\Omega^{2}} V\left(\phi_{1}, \phi_{2}\right) .
$$

Then

\footnotetext{
${ }^{8}$ As for the one-field case, we could use instead of $v$ an arbitrary mass scale.
}

$$
\begin{aligned}
\hat{L}= & \sqrt{\hat{g}}\left[-\frac{1}{2} v^{2} \hat{R}+\frac{1}{2} G_{i j} \partial_{\mu} \phi_{i} \partial^{\mu} \phi_{j}\right. \\
& \left.+\frac{q^{2}}{8} \frac{K}{\Omega} \omega_{\mu} \omega^{\mu}-\frac{q}{4} \omega^{\mu} \frac{K_{\mu}}{\Omega}-\hat{V}\right],
\end{aligned}
$$

where

$$
\begin{gathered}
G_{i j}=\frac{1}{6 v^{2} \Omega^{2}}\left(\begin{array}{cc}
\xi_{1}\left(1+\xi_{1}\right) \phi_{1}^{2}+\xi_{2} \phi_{2}^{2} & \xi_{1} \xi_{2} \phi_{1} \phi_{2} \\
\xi_{1} \xi_{2} \phi_{1} \phi_{2} & \xi_{2}\left(1+\xi_{2}\right) \phi_{2}^{2}+\xi_{1} \phi_{1}^{2}
\end{array}\right), \\
i, j=1,2 .
\end{gathered}
$$

The kinetic terms in $\hat{L}$ become diagonal (no mixing) in a new fields basis of $(\rho, \theta)$, where

$$
\begin{aligned}
& \phi_{1}=\frac{1}{\sqrt{1+\xi_{1}}} \rho \sin \theta, \\
& \phi_{2}=\frac{1}{\sqrt{1+\xi_{2}}} \rho \cos \theta .
\end{aligned}
$$

It is more illustrative, however, to first bring the Weyl terms in $\hat{L}$ to a quadratic form using

$$
\omega_{\mu}^{\prime}=\omega_{\mu}-\frac{1}{q} \partial_{\mu} \ln K,
$$

where we note that $K=\rho^{2}$. Adding $\hat{L}_{g}$ of Eq. (10) with Eq. (31) for the Weyl field $\omega_{\mu}$, then

$$
\begin{aligned}
\hat{L}+\hat{L}_{g}= & \sqrt{\hat{g}}\left[-\frac{1}{2} v^{2} \hat{R}+\frac{1}{2} G_{i j} \partial_{\mu} \phi_{i} \partial^{\mu} \phi_{j}-\frac{1}{8 K \Omega}\left(\partial_{\mu} K\right)^{2}\right. \\
& \left.-\frac{1}{4} F_{\mu \nu}^{\prime} F^{\prime \mu \nu}+\frac{K}{8 \Omega} q^{2} \omega_{\mu}^{\prime} \omega^{\prime \mu}-\hat{V}\right] \\
= & \sqrt{\hat{g}}\left[-\frac{1}{2} v^{2} \hat{R}+\frac{1}{2} T_{i j} \partial_{\mu} \phi_{i} \partial^{\mu} \phi_{j}-\frac{1}{4} F_{\mu \nu}^{\prime} F^{\prime \mu \nu}\right. \\
& \left.+\frac{K}{8 \Omega} q^{2} \omega_{\mu}^{\prime} \omega^{\prime \mu}-\hat{V}\right]
\end{aligned}
$$

where $F_{\mu \nu}^{\prime}=\tilde{D}_{\mu} \omega_{\nu}^{\prime}-\tilde{D}_{\nu} \omega_{\mu}^{\prime}$ is invariant under (37). Above we denoted $T_{i j}=G_{i j}+H_{i j}$, $(i, j=1,2)$, with

$H_{i j}=-\frac{1}{\Omega} \frac{1}{K}\left(\begin{array}{cc}\left(1+\xi_{1}\right)^{2} \phi_{1}^{2} & \left(1+\xi_{1}\right)\left(1+\xi_{2}\right) \phi_{1} \phi_{2} \\ \left(1+\xi_{1}\right)\left(1+\xi_{2}\right) \phi_{1} \phi_{2} & \left(1+\xi_{2}\right)^{2} \phi_{2}^{2}\end{array}\right)$.

In the new basis (36) the scalar kinetic terms in Eq. (38) are reduced to a single term and 


$$
\begin{aligned}
\hat{L}+\hat{L}_{g}= & \sqrt{\hat{g}}\left[\frac{-1}{2} v^{2} \hat{R}+\frac{1}{2} F(\theta) v^{2}\left(\partial_{\mu} \tan \theta\right)^{2}-\frac{1}{4} F_{\mu \nu}^{\prime} F^{\prime \mu \nu}\right. \\
& \left.+\frac{1}{2} m^{2}(\theta) \hat{g}^{\mu \nu} w_{\mu}^{\prime} w_{\nu}^{\prime}-\hat{V}\right]
\end{aligned}
$$

with

$F(\theta)=\frac{6 b}{\xi_{2}} \frac{\tan ^{2} \theta+\xi_{2} / \xi_{1}}{\left(1+\tan ^{2} \theta\right)\left(\tan ^{2} \theta+b\right)^{2}}, \quad b=\frac{\xi_{2}\left(1+\xi_{1}\right)}{\xi_{1}\left(1+\xi_{2}\right)}$.

Therefore, we are left with the angular kinetic term for $\theta$ only. The kinetic term of the radial (Goldstone) coordinate $\rho$ (where $\rho^{2}=K$ ) has disappeared, via the Stueckelberg mechanism, as it was eaten by the Weyl gauge boson $\omega^{\prime}$ in Eq. (37). This is similar to the case with one scalar field in Eq. (25). Thus, in the Einstein frame we have a massive vector boson and one (real) scalar field left $(\theta)$, while in the Jordan frame we had two (real) scalar fields and a massless $\omega_{\mu}$, so the number of d.o.f. is again conserved.

Further, the function $m^{2}(\theta)$ in (40) is given by

$m^{2}(\theta)=\frac{q^{2} K}{4 \Omega}=\frac{3 q^{2}}{2} \frac{v^{2}\left(1+\xi_{1}\right)\left(1+\xi_{2}\right)\left(1+\tan ^{2} \theta\right)}{\xi_{1}\left(1+\xi_{2}\right) \tan \theta^{2}+\xi_{2}\left(1+\xi_{1}\right)}$,

with

$$
v^{2}=\langle\rho\rangle^{2}\left[\frac{\xi_{1}}{1+\xi_{1}} \sin ^{2}\langle\theta\rangle+\frac{\xi_{2}}{1+\xi_{2}} \cos ^{2}\langle\theta\rangle\right] .
$$

Notice that if $\xi_{1}=\xi_{2}$ or if $\tan \theta$ is large, the function $m^{2}(\theta)$ is actually independent of $\theta$ and then the Weyl gauge field $\left(\omega_{\mu}^{\prime}\right)$ and the field $\theta$ decouple.

On the ground state $\theta=\langle\theta\rangle$ and the mass of $\omega_{\mu}$ is

$$
m^{2}(\langle\theta\rangle)=\frac{3}{2} q^{2}\langle\rho\rangle^{2} .
$$

The mass of $\omega_{\mu}$ is thus determined by $\langle\rho\rangle$ alone; unlike $\theta$ whose VEV is determined from $\hat{V}$ (see below), $\langle\rho\rangle$ cannot be predicted by the theory and is a free parameter (flat direction). ${ }^{9}$

The Planck scale $M_{p}^{2}=v^{2}$, Eq. (43), depends in general on $\langle\theta\rangle$. This is not a problem, since unlike $\rho$, the field variable $\theta$ does not change under a Weyl transformation, Eq. (1). However, if the theory has an $\mathcal{O}(2)$ symmetry, i.e., identical nonminimal couplings $\xi_{1}=\xi_{2}$, then $M_{p}$ is determined by the VEV of the dilaton alone $M_{p}^{2}=$ $v^{2}=\xi_{1}\langle\rho\rangle^{2} /\left(1+\xi_{1}\right)$; in this case, the would-be Goldstone

\footnotetext{
${ }^{9}\langle\rho\rangle$ may be fixed by quantum corrections; however, in quantum scale-invariant theories only ratios of field VEVs (scales) can be determined (in terms of dimensionless couplings), so it remains a free parameter.
}

(dilaton) field $\rho$ eaten by $\omega_{\mu}$ and fixing its mass also fixes the Planck scale. The same is true in the limit of large $\tan \theta \rightarrow \infty$, when $M_{p}^{2}=v^{2}=\xi_{1}\langle\rho\rangle^{2} /\left(1+\xi_{2}\right)$.

Regarding the potential $\hat{V}$ in Eq. (40), it is given by Eq. (33) expressed in terms of the new field variables $\rho, \theta$. With Eq. (7) and $V\left(\phi_{1}, \phi_{2}\right)$ the initial potential in the Jordan frame, then

$$
\hat{V}=36 v^{4} \frac{b^{2}}{\xi_{2}^{2}} \frac{V(c \tan \theta, 1)}{\left(\tan ^{2} \theta+b\right)^{2}}, \quad \text { where } c=\sqrt{\frac{1+\xi_{2}}{1+\xi_{1}}}
$$

which depends on $\theta$ only. Finally, another mechanism to decouple the dilaton was studied in [30] using a global version of the Weyl symmetry studied here (and assuming this survives black hole physics [42]).

\section{More fields and the Stueckelberg mechanism}

The Stueckelberg mechanism for $\omega_{\mu}$ can be extended for more scalar fields with nonminimal couplings, using general coordinates. For three fields $\phi_{1}=$ $\left(1 / \sqrt{1+\xi_{1}}\right) \rho \sin \theta \cos \zeta, \quad \phi_{2}=\left(1 / \sqrt{1+\xi_{2}}\right) \rho \sin \theta \sin \zeta$, and $\phi_{3}=\left(1 / \sqrt{1+\xi_{3}}\right) \rho \cos \theta$. As before, the kinetic term of radial field $\rho$ is the Goldstone eaten by the vector boson $\omega_{\mu}$ of mass $q^{2} K /\left.(4 \Omega)\right|_{\theta=\langle\theta\rangle}$. One is left with kinetic terms for the angular coordinates fields $\theta, \zeta$; similarly, the scalar potential will depend only on these fields. This generalization is useful in cases where one of the scalar fields left is a Higgs field, while the other is a second Higgs-like scalar, inflaton, etc. The scalar potential is then

$$
\begin{aligned}
\hat{V}(\theta, \phi) & =\frac{1}{\Omega^{2}} V\left(\phi_{1}, \phi_{2}, \phi_{3}\right) \\
& =\frac{36 v^{4} V\left(z_{1}, z_{2}, z_{3}\right)}{\left(\xi_{1} z_{1}^{2}+\xi_{2} z_{2}^{2}+\xi_{3} z_{3}^{2}\right)^{2}},
\end{aligned}
$$

where $V\left(\phi_{1}, \phi_{2}, \phi_{3}\right)$ is the initial potential in the Jordan frame and $z_{j}=\phi_{j} / \rho$ are functions of $\theta$ and $\zeta$ only. If $\xi_{1}=\xi_{2}=\xi_{3}$, then the Planck scale is also determined by the same $\rho$ field. The extension to more scalar fields is straightforward. This study can also be extended to include additional (Weyl gauge-invariant) terms quadratic in the scalar curvature $[34,35]$.

\section{Other implications: UV scale-invariant regularization}

The above results have implications for models with (global) scale invariance at the quantum level. Such models are important since they can have a quantum stable hierarchy between two scalar fields' VEVs (Higgs and dilaton), which is relevant for the SM hierarchy problem, as we detail below.

Consider first a classical scale-invariant model. The SM with a vanishing Higgs mass parameter is an example. This 
symmetry can be preserved at the quantum level, by ensuring that the UV regularization respects it. This is done by replacing the subtraction scale $\mu$ by the dilaton field $\rho$ [2]. After spontaneous breaking of this symmetry, $\mu \sim\langle\rho\rangle$. In this way one obtains scale-invariant results at the quantum level $[3-6,10]$. After the quantum calculation, one can expand the result (e.g., the scalar potential) about the VEV of the dilaton to recover standard results (e.g., Coleman-Weinberg potential), plus additional higher dimensional operators suppressed by the dilaton VEV [6]. Such models have only spontaneous breaking of the scale symmetry, thus there is no dilatation anomaly $[2,4,7,10]$.

The relation to the hierarchy problem is that the dilaton VEV is fixing $M_{p}$ and so it must be much higher than the Higgs VEV. Such hierarchy can be the result of one initial classical tuning of the (dimensionless) couplings. This tuning remains stable at the quantum level, due to quantum scale invariance and a shift symmetry of the dilaton (Goldstone mode) [9]. However, the dilaton remains in the spectrum as a flat direction, even at quantum level. One can then ask what happens to this flat direction for a more general, local Weyl symmetry.

The result of this paper answers this question. As we saw, the dilaton is eaten by the Weyl field $\omega_{\mu}$, which becomes massive, decouples from the spectrum and leaves a potential function of the angular variables' fields only (which can be the Higgs field, inflaton, etc.).

For example, in a two-field case and assuming a potential $V$ of Eq. (30), after decoupling the potential $\hat{V}$ depends only on $h \equiv v \cot \theta$, which can be the neutral Higgs field; taking for simplicity $\xi_{2}=0$ and large $\xi_{1}$, after dilaton decoupling, the scalar potential (for a canonical kinetic term for $h$ ) becomes in the Einstein frame

$$
\hat{V}=\frac{\lambda_{2}}{4 !}\left(h^{2}-\frac{\sqrt{\lambda_{1}}}{\sqrt{\lambda_{2}}} \frac{v^{2}}{\left(\xi_{1} / 6\right)}\right)^{2},
$$

which is indeed that of the SM, with $m_{h}^{2}=\sqrt{\lambda_{1} \lambda_{2}} v^{2} / \xi_{1} \ll$ $v^{2} \sim\langle\rho\rangle^{2}$ for small (ultraweak) couplings and large $\xi_{1}$. What happens with this hierarchy at the quantum level?

Before the Stueckelberg mechanism, the dilaton can enforce a scale- (or Weyl-) invariant UV regularization [2] of the quantum corrections to potential (30), as described above. In this way one can construct a quantum scale(Weyl-) invariant theory, dilatation (conformal) anomalyfree, respectively. In this case, the mentioned classical hierarchy between $m_{h}^{2}$ and $v^{2} \sim\langle\rho\rangle^{2}$ remains stable at the quantum level (for more details see discussion in $[6,10]$ ).

\section{INFLATION FROM WEYL GAUGE SYMMETRY}

In this section, we study inflation in models with spontaneously broken Weyl gauge symmetry. We consider the case of the two scalar fields of Sec. III B and regard the potential for the angular-variable field $\theta$, obtained after the Stueckelberg mechanism, as being responsible for inflation. The potential becomes constant at large $\tan \theta$. In this limit, from Eqs. (40) and (42), $\theta$ and $\omega_{\mu}^{\prime}$ decouple and the action for the inflaton $(\theta)$ can be written as

$$
\begin{aligned}
L_{\text {infl }}= & \sqrt{\hat{g}}\left[-\frac{1}{2} v^{2} \hat{R}+\frac{3 b v^{2}}{\xi_{2}} \frac{\tau^{2}+\xi_{2} / \xi_{1}}{\left(1+\tau^{2}\right)\left(\tau^{2}+b\right)^{2}}\left(\partial_{\mu} \tau\right)^{2}-\hat{V}(\tau)\right], \\
\tau & \equiv \tan \theta
\end{aligned}
$$

For $V$ of (29), the Einstein frame potential expressed in terms of $\tau$ is

$$
\begin{aligned}
\hat{V} & =\frac{36 v^{4}}{\left(\xi_{1} \phi_{1}^{2}+\xi_{2} \phi_{2}^{2}\right)^{2}}\left(\frac{\lambda_{1}}{4 !} \phi_{1}^{4}+\frac{\lambda_{12}}{4} \phi_{1}^{2} \phi_{2}^{2}+\frac{\lambda_{2}}{4 !} \phi_{2}^{4}\right) \\
& =\frac{36 v^{4}\left(1+\xi_{1}\right)^{2}}{\xi_{1}^{2}\left(\tau^{2}+b\right)^{2}}\left(c_{1} \tau^{4}+c_{12} \tau^{2}+c_{2}\right),
\end{aligned}
$$

with

$$
\begin{array}{ll}
c_{1} & =\frac{\lambda_{1}}{4 !\left(1+\xi_{1}\right)^{2}}, \quad c_{12}=\frac{\lambda_{12}}{4\left(1+\xi_{1}\right)\left(1+\xi_{2}\right)}, \\
c_{2} & =\frac{\lambda_{2}}{4 !\left(1+\xi_{2}\right)^{2}} .
\end{array}
$$

The potential is similar to that in Higgs portal inflation $[41,43]$, but in our case the angular field $\theta$ is the dynamical field responsible for a slow-roll inflation (instead of being frozen). For the case of global Weyl invariant models, inflation was already studied in $[31,36]$. Note that if $c_{12}=$ $-2 \sqrt{c_{1} c_{2}}\left(c_{1,2}>0\right)$, then $\hat{V}=0$ at the minimum.

In the following, we consider the case $\xi_{1}=\xi_{2}$, which is preferable since then $\theta$ and $\omega_{\mu}^{\prime}$ are decoupled from each other for all values of $\theta$ [cf. (40)]. Then $b=1$, and the kinetic term acquires a canonical form $(1 / 2)\left(\partial_{\mu} \chi\right)^{2}$, with the actual inflaton field $\chi$ defined by

$$
\chi=v \theta \sqrt{\frac{6}{\xi_{1}}} .
$$

Then the inflaton potential in Eq. (49) becomes

$$
\begin{aligned}
\hat{V} & =V_{0} \cos ^{4} \theta\left(\tan ^{4} \theta+\frac{c_{12}}{c_{1}} \tan ^{2} \theta+\frac{c_{2}}{c_{1}}\right), \text { with } \\
V_{0} & =\frac{36 v^{4}}{\xi_{1}^{2}} c_{1}\left(1+\xi_{1}\right)^{2},
\end{aligned}
$$

with $\theta \equiv \sqrt{\xi_{1} / 6} \chi / v$. The potential is illustrated in Fig. 1 for some choices of the quartic couplings that give $\hat{V}=0$ at the minimum. Inflation takes place at $\tan \theta \gg 1\left(\right.$ or $\theta \sim \frac{\pi}{2}$ ) for which $V=V_{0}\left[1+\left(c_{12} / c_{1}-2\right) \cot ^{2} \theta+\left(3-2 c_{12} / c_{1}+\right.\right.$ $\left.\left.c_{2} / c_{1}\right) \cot ^{4} \theta+\cdots\right]$. For $\xi_{1} \neq \xi_{2}$ with $\tan \theta \gg 1$, we note 

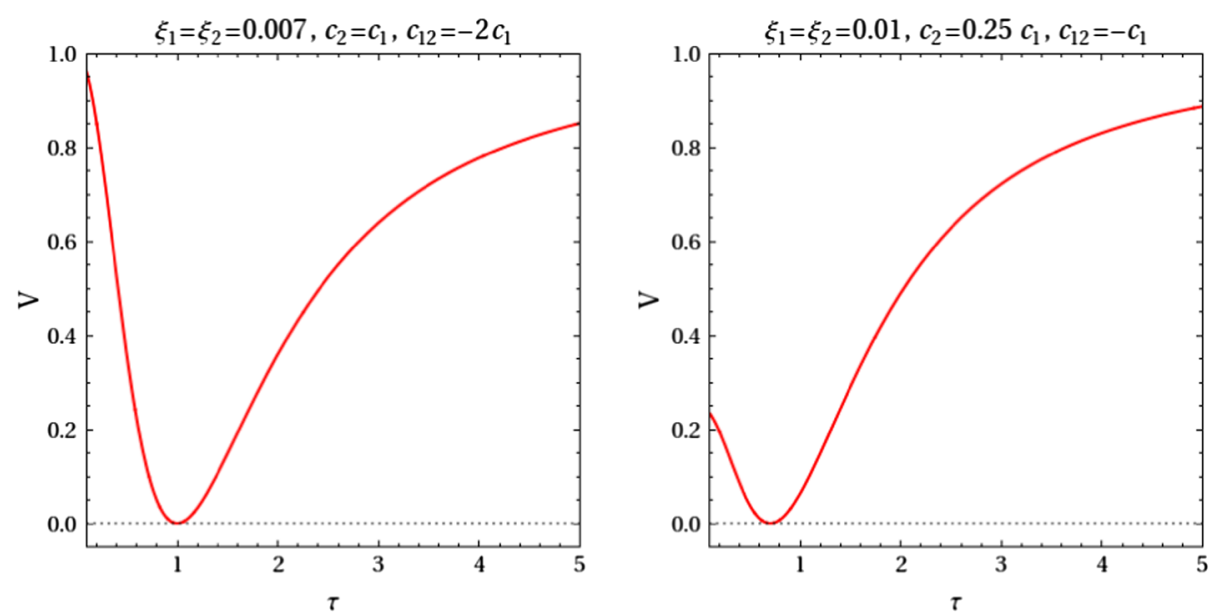

FIG. 1. Inflaton potential as a function of $\tau=\tan \theta$, for $\xi_{1}=\xi_{2}$ and ratios $c_{1,2} / c_{1}, c_{2} / c_{1}$ of values shown above. $V=0$ at the minimum [note $\theta=(\chi / v) \sqrt{\xi_{1} / 6}$ with $\chi$ the actual inflaton]. The values of the spectral index and tensor-to-scalar ratio for these two cases are shown in Fig. 2.
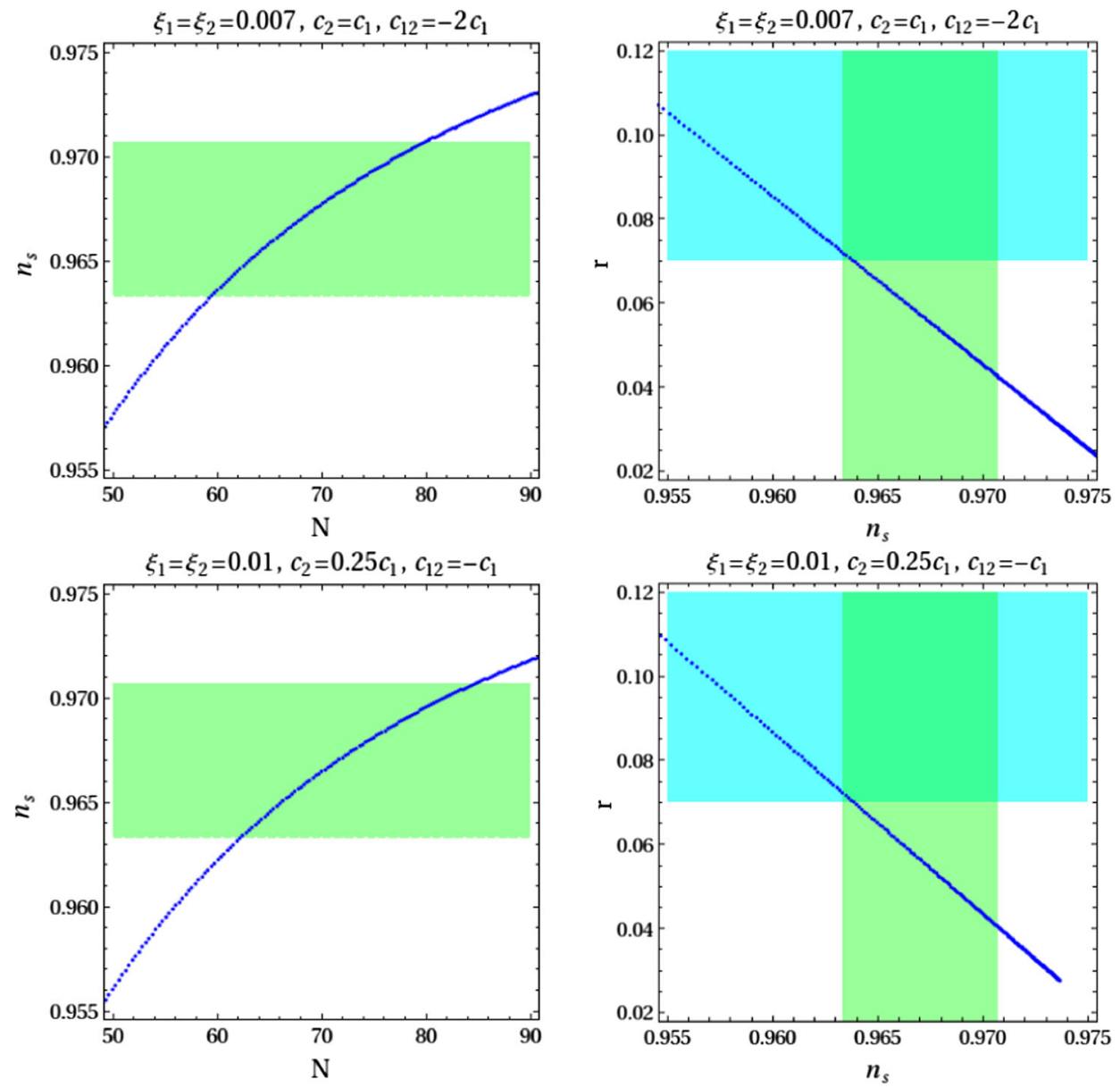

FIG. 2. (Left) The spectral index $n_{s}$ as a function of the number of $e$-foldings $N$ for two different cases. (Right) The spectral index $n_{s}$ versus tensor-to-scalar ratio $r$ for the corresponding cases. The green region in all the plots corresponds to Planck 2018 values for $n_{s}$ within $1 \sigma$ (see text). The cyan region with $r>0.07$ is excluded by Planck at $95 \%$ C.L. We chose $\xi_{1}=\xi_{2}$ for all the plots and $c_{2} / c_{1}=1$ and $c_{12} / c_{1}=(-2)$ (upper) and $c_{2} / c_{1}=0.25$ and $c_{12} / c_{1}=(-1)$ (lower). Here $c_{12}$ is fixed by the condition $\hat{V}=0$ at the minimum. 
that there is an approximate relation between $\theta$ and the canonical inflaton field $\chi$ similar to that in Eq. (51).

The slow-roll parameters (for $\xi_{1}=\xi_{2}$ ) are given by

$\epsilon=\frac{1}{3} \xi_{1} \frac{\left(c_{2}-c_{1}+\left(c_{1}-c_{12}+c_{2}\right) \cos (2 \theta)\right)^{2} \sec ^{4} \theta \tan ^{2} \theta}{\left(c_{2}+c_{12} \tan ^{2} \theta+c_{1} \tan ^{4} \theta\right)^{2}}$,

$\eta=-\frac{1}{3} \xi_{1} \frac{\left(\left(c_{2}-c_{1}\right) \cos (2 \theta)+\left(c_{1}-c_{12}+c_{2}\right) \cos (4 \theta)\right) \sec ^{4} \theta}{c_{2}+c_{12} \tan ^{2} \theta+c_{1} \tan ^{4} \theta}$.

We find that $\epsilon$ and $\eta$ can be small simultaneously for $\xi_{1} \ll 1$.

If we choose $c_{2}=c_{1}, c_{12}=-2 c_{1}$ then the expressions of the slow-roll parameters simplify further,

$$
\begin{aligned}
\epsilon & =\frac{4}{3} \xi_{1} \tan ^{2}(2 \theta), \\
\eta & =\frac{4}{3} \xi_{1}\left(-1+\tan ^{2}(2 \theta)\right),
\end{aligned}
$$

which leads to the scalar spectral index and the tensor-toscalar ratio as

$$
n_{s}=1+2 \eta_{*}-6 \epsilon_{*}=1-\frac{8}{3} \xi_{1}\left(1+2 \tan ^{2}\left(2 \theta_{*}\right)\right),
$$

and

$$
r=16 \epsilon_{*}=\frac{64}{3} \xi_{1} \tan ^{2}\left(2 \theta_{*}\right) .
$$

Further, the number of $e$-foldings during inflation is also given by

$$
N=v^{-1} \int_{\chi_{\text {end }}}^{\chi_{*}} \frac{\operatorname{sign}\left(\hat{V}^{\prime}\right) d \chi}{\sqrt{2 \epsilon(\chi)}}=\frac{3}{4 \xi_{1}} \log \left|\frac{\sin \left(2 \theta_{\text {end }}\right)}{\sin \left(2 \theta_{*}\right)}\right|,
$$

where $\theta_{*}$ is evaluated at the horizon exit and $\theta_{\text {end }}$ is the inflaton value at the end of inflation. Inflation ends at $\epsilon=1$, i.e., $\left|\tan \left(2 \theta_{\text {end }}\right)\right|=\left(\frac{3}{4 \xi_{1}}\right)^{1 / 2}$ from Eq. (55).

The normalization of the cosmic microwave background anisotropies, $V_{0} /\left(24 \pi^{2} v^{4} \epsilon_{*}\right)=2.1 \times 10^{-9}$ [44], constrains $c_{1}$ (or, equivalently, the quartic coupling $\lambda_{1}$ ) and the nonminimal couplings $\xi_{1,2}$ to satisfy

$$
\frac{c_{1}\left(1+\xi_{1}\right)^{2}}{\xi_{1}^{3}}=1.8 \times 10^{-8} \tan ^{2}\left(2 \theta_{*}\right) .
$$

This constraint is respected by choosing small values of $c_{1}$ (or $\lambda_{1}$ ), for given $\xi_{1}$.

We have that $n_{s}=0.9670 \pm 0.0039 \quad(68 \%$ C.L. $)$ and $r<0.07$ (95\% C.L.) from Planck2018 (TT, TE, $\mathrm{EE}+$ low $\mathrm{E}+$ lensing + BK14 + BAO) [44]. In Fig. 2, we illustrate the relation between the spectral index versus the number of $e$-foldings in the left plots and showed the spectral index versus the tensor-to-scalar ratio in the right plots. Here we have fixed $\xi_{1}=\xi_{2}=0.007, c_{2}=c_{1}$, and $c_{12}=-2 c_{1}$ in the upper panel, and $\xi_{1}=\xi_{2}=0.01$, $c_{2}=0.25 c_{1}$, and $c_{12}=-c_{1}$ in the lower panel. As a result, we find that our model of inflation is consistent with the observed spectral index and the bound on $r$, for small nonminimal couplings and appropriate quartic couplings.

\section{CONCLUSIONS}

In this work, we discussed the Weyl conformal symmetry and its spontaneous breaking and some implications for model building beyond the SM and inflation.

In models with conformal symmetry (of the BransDicke-Jordan type) with scalar fields with nonminimal couplings to the Ricci scalar, one can generate spontaneously the Planck scale from the VEV of a scalar field (or a combination of them). However, a positive (negative) Newton constant is accompanied by a negative (positive) kinetic term for this field, respectively. This situation is naturally avoided in models with an additional Weyl gauge symmetry and a gauge field $\omega_{\mu}$ that is of geometric origin, with a gauge transformation dictated by the conformal transformation of the metric.

We showed that the Weyl field $\omega_{\mu}$ couples only to the scalar sector but not to the fermionic sector of a SM-like Lagrangian in curved space-time, which is interesting for model building. Further, the field $\omega_{\mu}$ undergoes a Stueckelberg mechanism and becomes massive after eating the radial mode $\rho \sim \sqrt{K}$ (in field space) and would-be Goldstone mode (dilaton). The Weyl gauge symmetry is then spontaneously broken (and there are no negative kinetic terms in the theory). Further, the VEV $\langle\rho\rangle$ determines the mass of $\omega_{\mu}$ and the Planck scale $M_{p}$ (up to possible additional angular variables' field dependence). The mass of $\omega_{\mu}$ can be larger or smaller than $M_{p}$ depending on the scalar field's charge and nonminimal couplings. After decoupling of $\omega_{\mu}$ the potential depends on the angular variables' fields only, which can play the role of the neutral Higgs field, inflaton, etc. For two scalar fields of equal nonminimal couplings, the field $\omega_{\mu}$ decouples from the action even if it is light.

For the case with two scalar fields, the scalar potential generally depends only on the angular-variable field $\theta$, and it is nearly constant at large $\tan \theta$, when $\omega_{\mu}$ also decouples. Therefore, the potential can be relevant for a single-field inflation. Investigating the details of the inflaton potential, we found that successful inflation is possible, with values of $n_{s}$ and $r$ consistent with Planck2018 constraints, for perturbative values of the couplings.

While this study was formulated in (pseudo)Riemannian geometry extended with a real Weyl field (undergoing a gauge transformation dictated by conformal transformation of the metric), the natural framework is that of Weyl 
conformal geometry where this symmetry is manifest. In the Riemannian case, imposing this symmetry avoids the ghost kinetic term of conformal theory and leads to a Lagrangian with a current $\partial_{\mu} K$ that interacts with the Weyl field. This Lagrangian was shown to be identical, up to a total derivative term, to that obtained in Weyl geometry where the Weyl-symmetric Lagrangian is naturally built in, with curvature scalar, tensors, and affine connection of Weyl geometry. This equivalence is shown for a SM-like Lagrangian endowed with Weyl gauge symmetry, using the relation between $R$ computed in Riemann geometry with Levi-Civita connection and its counterpart in Weyl geometry. This Lagrangian can be used for examining the phenomenological constraints on the SM extended with Weyl gauge symmetry.

\section{ACKNOWLEDGMENTS}

The authors are grateful for hospitality and support from CERN Theory Division where this work was done. The work of H.M.L. is supported in part by the Basic Science Research Program through the National Research Foundation of Korea (NRF) funded by the Ministry of Education, Science and Technology (NRF2016R1A2B4008759 and NRF-2018R1A4A1025334). D. M. G. is supported by National Programme PN 18090101.
[1] W. A. Bardeen, On naturalness in the standard model, 1995 Ontake Summer Institute, Ontake Mountain, Japan, 1995, Fermilab Report No. FERMILAB-CONF-95-391-T, 1995.

[2] F. Englert, C. Truffin, and R. Gastmans, Conformal invariance in quantum gravity, Nucl. Phys. B117, 407 (1976).

[3] M. Shaposhnikov and D. Zenhausern, Quantum scale invariance, cosmological constant and hierarchy problem, Phys. Lett. B 671, 162 (2009).

[4] R. Armillis, A. Monin, and M. Shaposhnikov, Spontaneously broken conformal symmetry: Dealing with the trace anomaly, J. High Energy Phys. 10 (2013) 030.

[5] F. Gretsch and A. Monin, Perturbative conformal symmetry and dilaton, Phys. Rev. D 92, 045036 (2015).

[6] D. M. Ghilencea, Quantum implications of a scale invariant regularization, Phys. Rev. D 97, 075015 (2018); Manifestly scale-invariant regularization and quantum effective operators, Phys. Rev. D 93, 105006 (2016); D. M. Ghilencea, Z. Lalak, and P. Olszewski, Two-loop scale-invariant scalar potential and quantum effective operators, Eur. Phys. J. C 76, 656 (2016).

[7] C. Tamarit, Running couplings with a vanishing scale anomaly, J. High Energy Phys. 12 (2013) 098.

[8] P. G. Ferreira, C. T. Hill, and G. G. Ross, Scale-independent inflation and hierarchy generation, Phys. Lett. B 763, 174 (2016).

[9] R. Foot, A. Kobakhidze, K. L. McDonald, and R. R. Volkas, Poincaré protection for a natural electroweak scale, Phys. Rev. D 89, 115018 (2014).

[10] D. M. Ghilencea, Z. Lalak, and P. Olszewski, Standard model with spontaneously broken quantum scale invariance, Phys. Rev. D 96, 055034 (2017).

[11] K. Allison, C. T. Hill, and G. G. Ross, Ultra-weak sector, Higgs boson mass, and the dilaton, Phys. Lett. B 738, 191 (2014).

[12] E. J. Chun, S. Jung, and H. M. Lee, Radiative generation of the Higgs potential, Phys. Lett. B 725, 158 (2013); Erratum 730, 357(E) (2014).
[13] C. Brans and R.H. Dicke, Mach's principle and a relativistic theory of gravitation, Phys. Rev. 124, 925 (1961); R. H. Dicke, Mach's principle and invariance under transformation of units, Phys. Rev. 125, 2163 (1962); P. Jordan, Schwerkraft und Weltall 2nd revised ed. (Vieweg, Braunschweig, 1955).

[14] H. Weyl, Gravitation und elektrizität, Sitzungsberichte der Königlich Preussischen Akademie der Wissenschaften zu Berlin (1918), pp. 465-480. For a recent review and references see [15].

[15] E. Scholz, Weyl geometry in late 20th century physics, arXiv:1111.3220; The unexpected resurgence of Weyl geometry in late 20-th century physics, Einstein Stud. 14, 261 (2018); Paving the way for transitions: A case for Weyl geometry, Einstein Stud. 13, 171 (2017).

[16] P. A. M. Dirac, Long range forces and broken symmetries, Proc. R. Soc. A 333, 403 (1973).

[17] E. Scholz, Higgs and gravitational scalar fields together induce Weyl gauge, Gen. Relativ. Gravit. 47, 7 (2015).

[18] I. Bars, P. Steinhardt, and N. Turok, Local conformal symmetry in physics and cosmology, Phys. Rev. D 89, 043515 (2014).

[19] L. Smolin, Towards a theory of space-time structure at very short distances, Nucl. Phys. B160, 253 (1979).

[20] H. Cheng, The Possible Existence of Weyl's Vector Meson, Phys. Rev. Lett. 61, 2182 (1988).

[21] H. Nishino and S. Rajpoot, Implication of compensator field and local scale invariance in the standard model, Phys. Rev. D 79, 125025 (2009).

[22] M. de Cesare, J. W. Moffat, and M. Sakellariadou, Local conformal symmetry in non-Riemannian geometry and the origin of physical scales, Eur. Phys. J. C 77, 605 (2017).

[23] H. C. Ohanian, Weyl gauge-vector and complex dilaton scalar for conformal symmetry and its breaking, Gen. Relativ. Gravit. 48, 25 (2016).

[24] Y. Tang and Y. L. Wu, Inflation in gauge theory of gravity with local scaling symmetry and quantum induced symmetry breaking, Phys. Lett. B 784, 163 (2018). 
[25] A. Davidson and T. Ygael, Frozen up dilaton and the GUT/Planck mass ratio, Phys. Lett. B 772, 5 (2017).

[26] J. W. Moffat, Scalar-tensor-vector gravity theory, J. Cosmol. Astropart. Phys. 03 (2006) 004.

[27] L. Heisenberg, Scalar-vector-tensor gravity theories, J. Cosmol. Astropart. Phys. 10 (2018) 054.

[28] J. T. Wheeler, Weyl geometry, Gen. Relativ. Gravit. 50, 80 (2018).

[29] I. Quiros, Scale invariant theory of gravity and the standard model of particles, arXiv:1401.2643.

[30] P. G. Ferreira, C. T. Hill, and G. G. Ross, No fifth force in a scale invariant universe, Phys. Rev. D 95, 064038 (2017).

[31] P. G. Ferreira, C. T. Hill, and G. G. Ross, Weyl current, scale-invariant inflation and Planck scale generation, Phys. Rev. D 95, 043507 (2017).

[32] P. G. Ferreira, C. T. Hill, and G. G. Ross, Inertial spontaneous symmetry breaking and quantum scale invariance, arXiv:1801.07676; C. T. Hill, Inertial symmetry breaking, arXiv:1803.06994.

[33] B. Holdom, Two U(1)'s and epsilon charge shifts, Phys. Lett. B 166, 196 (1986).

[34] D. M. Ghilencea, Spontaneous breaking of Weyl quadratic gravity to Einstein action and Higgs potential, J. High Energy Phys. 03 (2019) 049.

[35] D. M. Ghilencea, Stueckelberg breaking of Weyl conformal geometry and applications to gravity, arXiv: 1904.06596.
[36] J. Garcia-Bellido, J. Rubio, M. Shaposhnikov, and D. Zenhausern, Higgs-dilaton cosmology: From the early to the late universe, Phys. Rev. D 84, 123504 (2011).

[37] G. K. Karananas and J. Rubio, On the geometrical interpretation of scale-invariant models of inflation, Phys. Lett. B 761, 223 (2016).

[38] S. Casas, M. Pauly, and J. Rubio, Higgs-dilaton cosmology: An inflation-dark-energy connection and forecasts for future galaxy surveys, Phys. Rev. D 97, 043520 (2018).

[39] K. Sravan Kumar and P. Vargas Moniz, Conformal GUT inflation, proton lifetime and non-thermal leptogenesis, arXiv:1806.09032.

[40] A. S. Koshelev, K. Sravan Kumar, and P. Vargas Moniz, Effective models of inflation from a nonlocal framework, Phys. Rev. D 96, 103503 (2017).

[41] F. Bezrukov, G. K. Karananas, J. Rubio, and M. Shaposhnikov, Higgs-dilaton cosmology: An effective field theory approach, Phys. Rev. D 87, 096001 (2013).

[42] T. Banks, M. Johnson, and A. Shomer, A note on gauge theories coupled to gravity, J. High Energy Phys. 09 (2006) 049.

[43] O. Lebedev and H. M. Lee, Higgs portal inflation, Eur. Phys. J. C 71, 1821 (2011).

[44] Y. Akrami et al. (Planck Collaboration), Planck 2018 results. X. Constraints on inflation, arXiv:1807.06211. 